\title{
On Mode Selection and Power Control for Uplink D2D Communication in Cellular Networks
}

\author{
Konpal Shaukat Ali, Hesham ElSawy, and Mohamed-Slim Alouini \\ Computer, Electrical and Mathematical Sciences and Engineering (CEMSE) Division \\ King Abdullah University of Science and Technology, KAUST \\ Thuwal, Makkah Province, Saudi Arabia \\ \{konpal.ali, hesham.elsawy, slim.alouini\}@kaust.edu.sa
}

\begin{abstract}
Device-to-device (D2D) communication enables users lying in close proximity to bypass the cellular base station (BS) and transmit to one another directly. This offloads traffic from the cellular network, improves spatial frequency reuse and energy efficiency in the network. We present a comprehensive and tractable analytical framework for D2D-enabled uplink cellular networks with two different flexible mode-selection schemes. The power-control cutoff thresholds of the two communication modes have been decoupled unlike past work on the subject. We find that for a given network, an optimal value exists not only for the biased mode selection criterion, but also for $r$, the ratio of the power-control cutoff thresholds of the two communication modes, which maximizes spatial spectral efficiency. Also, $r$ turns out to be a more robust parameter for optimizing network performance. Further, it is shown that the second scheme, which prioritizes spatial frequency reuse over the per-user achievable performance compared to the first scheme, achieves almost the same overall network performance; thereby trading per user performance to serve a larger number of users.
\end{abstract}

\section{INTRODUCTION}

Recently, the concept of device-to-device (D2D) communication has emerged to cater the ever-increasing demand of data rate and system capacity. D2D enables users equipment (UEs) lying in close proximity, that intend to exchange information, to bypass the cellular base station (BS) and communicate in a peer-to-peer fashion. It is envisioned that D2D communication between UEs can improve the network performance in terms of spatial frequency reuse, latency, and energy consumption [1]. In other words, D2D communication replaces the conventional two-hop cellular link by a short-range, low-power, and direct D2D link which may improve the latency and power consumption. Further, while the conventional cellular association dictates single link per channel per cell, D2D links can potentially reuse the same channel over the spatial domain with no restriction over the cell boundaries, which increases the spatial frequency reuse. However, the envisioned D2D gains come at the expense of more complicated network management. Choosing the mode of operation (i.e. cellular mode or D2D mode) and neighbor discovery are two new network functions that arise with D2D communication. Further, interference management between cellular and D2D links also needs to be taken into account.

For efficient network operation, it is fundamental to understand and characterize the performance of D2D enabled cellular networks. In this paper, we study the effect of enabling
D2D communication in a cellular network. More particularly, we develop an analytical framework to model Signalto-Interference-Noise-Ratio (SINR) in D2D enabled cellular networks. Our main performance metrics are the outage probability, defined as the probability that the SINR falls below a predefined threshold $\theta$, and the ergodic rate, defined by the seminal Shannon capacity formula; i.e. we investigate the effect of D2D interference on the cellular outage probability and ergodic rate. We also examine the outage probability and the achieved ergodic rate of the D2D users. The developed analytical paradigm is then used to obtain design insights for the mode selection and power control.

The developed analytical framework is based on the stochastic geometry toolset, which is a powerful tool to model random networks. The motivation to exploit stochastic geometry is two fold. Firstly, the locations of the users are random and D2D communication imposes random ad-hoc links to the cellular infrastructure. Secondly, cellular networks deviate from the traditional hexagonal grid model and exhibit random topologies [2]-[4]. To this end, for analytical tractability, the Poisson Point Process (PPP) is used to model the network topology and the spatial distribution of the users. The PPP has been shown to lead to a tight lower bound on the network performance [2]-[6].

This work comes in line with the several efforts invested in the literature to model D2D communication in cellular networks [7]-[12]. The authors of [7] ensure SINR violation for cellular users due to D2D interferers is kept below a threshold, using a simple power control method. The analysis, however, is limited to one user, one cell, and one D2D link. In [8], given an interference threshold to the cellular network, the maximum intensity of D2D devices possible in an uplink cellular network is found. The optimal intensity and transmit power, that maximize capacity for a D2D enabled two-tier uplink network with outage probability constraints is found in [9]. Mode selection and power control are not considered in [8] and [9], and the D2D link distances are assumed to be fixed. D2D distance-based mode selection criteria has been employed in [10] and [11]. A biased distance-based mode selection scheme for underlay is considered in [12], which gives the flexibility to control the amount of traffic offloaded from the cellular to the D2D mode.

The system model studied in this paper is similar to the 
one in [12]. However, we investigate the effect of allowing decoupled power-control cutoff threshold for the D2D and cellular users, and employ two different D2D mode selection schemes. We also discuss the performance of underlay and overlay operation of the D2D users. To this end, we show that not only does there exist an optimal value of the D2D biasing factor, but an optimal ratio between the decoupled power-control cutoff thresholds also exists that maximizes spatial spectral efficiency. Also, we show that the second mode selection scheme, which prioritizes spatial frequency reuse over the per user achievable performance, still achieves almost the same overall network performance, in terms of outage and network spectral efficiency, as the first scheme. Hence employing the first scheme allows higher quality per user performance for fewer users, while the second scheme allows us to trade per user performance to serve a larger number of users.

The rest of the paper is organized as follows: the network model and the two mode selection schemes are described in Section II. The statistics of the the transmit powers, the assumptions made, the SINR Analysis, and the performance metrics being used are covered in Section III. Section IV contains the results and analysis for the underlay and briefly for the overlay case. Section V concludes the paper.

\section{System Model}

\section{A. Network Model}

We consider a single-tier uplink network. The BSs and UEs are distributed in $\mathbb{R}^{2}$ via independent PPPs $\Psi$ and $\Phi$, with intensities $\lambda$ and $\mathcal{U}$, respectively. We assume a congested network $(\mathcal{U} \gg \lambda)$, so that each cellular BS always has at least one UE transmitting to it. D2D communication is enabled, hence users that have their receiver located within the D2Dproximity can bypass the BS and communicate directly via the D2D mode. The D2D-proximity is defined as the area centered at the transmitting UE, with radius $R_{\max }=\left(P_{u} / \rho_{\min }\right)^{1 / \eta_{d}}$, where $P_{u}$ is the maximum transmit power of a UE, $\rho_{\min }$ the minimum power the receiving UE is able to recover (receiversensitivity) and $\eta_{d}$ is the path loss exponent for D2D links. A UE that has its receiver within the D2D proximity is referred to as a potential D2D UE. The intensity of potential D2D UEs is designated by $\mathcal{D}$. Note, potential D2D UEs do not necessarily transmit in the D2D mode, instead, they select their mode of operation (i.e. cellular/D2D mode) according to the mode selection criterion presented later in this section.

We assume potential D2D UEs have their receivers located at a distance $r_{d}$, with probability density function (PDF) $f_{r_{d}}(x)=1 / R_{\max }, 0 \leq x \leq R_{\max }$. Due to the PPP assumption, the distance between a BS and its transmitting UE, $r_{c}$, follows a Rayleigh distribution; $f_{r_{c}}(x)=2 \pi \lambda r e^{-\pi \lambda x^{2}}, x \geq 0$. We consider a power-law path-loss model; since the propagation environments for the cellular and D2D links may be significantly different, different path loss exponents $\eta_{c}>2$ and $\eta_{d}>2$ are used for modeling the cellular and D2D links respectively. Hence, the power decays at the rate $x^{-\eta_{c}}$ $\left(x^{-\eta_{d}}\right)$ for the cellular (D2D) links, where $x$ is the propagation distance. A Rayleigh fading environment is considered and the channel gains are assumed to be independent of the locations of the transmitters, receivers, and independent of one another. The channel power gain, $h$, is modeled by a unit mean exponential random variable (RV).

Users associate to BSs based on the radio signal strength (RSS), which reduces to the nearest BS association in singletier networks. Due to the limited transmit power of the UEs, a truncated channel inversion power control is employed. That is, only users that can compensate for the path loss and maintain a predefined average power level at their receivers are allowed to transmit. Unlike previous work in [12], the cutoff threshold for the power control of the two communication modes has been segregated into $\rho_{d}$ for D2D receivers and $\rho_{c}$ for cellular BSs. This has been done to decouple the D2D and cellular power control which may lead to an enhanced network pefromance. For the sake of simple presentation, we define $r=\rho_{c} / \rho_{d}$. A D2D connection (cellular connection) can be established if the power required to achieve $\rho_{d}\left(\rho_{c}\right)$ at the receiving UE (base station) does not exceed $P_{u}$. For users in the cellular mode, if the transmit-power required to achieve $\rho_{c}$ exceeds $P_{u}$, the UE does not transmit and goes into outage, denoted as cellular-truncation outage. Due to the PPP assumption, this cellular outage probability is derived to be $\mathcal{O}_{p}=e^{-\pi \lambda\left(P_{u} / \rho_{c}\right)^{\frac{2}{\eta_{c}}}}$, and UEs in cellular outage are said to not be covered by the BS. For the D2D users, if the transmit-power required to achieve $\rho_{d}$ exceeds $P_{u}$, we have the following two truncation schemes:

- Scheme 1: the UE in truncation does not transmit and goes into D2D-truncation outage.

- Scheme 2: the UE in truncation transmits with power $P_{u}$ if an interference constraint at the cellular UEs is satisfied, otherwise it goes into D2D-truncation outage.

It is worth noting that $\rho_{d}$ controls the non-truncation D2Dproximity as $R=\left(P_{u} / \rho_{d}\right)^{1 / \eta_{d}}$; thus the intensity of the potential D2D UEs that are not in truncation is $p \mathcal{D}$ and the D2D truncation probability is given by $(1-p)$, where $p=R / R_{\max }$.

We assume universal frequency reuse across the entire network, but that BSs assign unique channels to each of their associated UEs and so there is no intracell interference between cellular users. We focus on one uplink channel which is shared by the D2D and cellular UEs in the underlay case. In the overlay case, we consider the uplink channel to be partitioned into a fraction $\tau$ for the D2D UEs and $1-\tau$ for the cellular UEs. Hence, the overlay prohibits cross-mode interference.

\section{B. Mode Selection and UE Classification}

We consider two flexible mode selection schemes; the flexibility comes from the fact that a biased mode-selection criteria, that provides Interference Protection (IP) to the cellular UEs from the D2D communication mode, is employed. The IP criteria is that potential D2D UEs can select the D2D mode only if $r_{d}^{\eta_{d}} \rho_{d} \leq T_{d} r_{c}^{\eta_{c}} \rho_{c}$, where $T_{d}$ is the bias factor used to 


\begin{tabular}{|c|c|c|c|c|c|c|c|c|}
\hline Scheme & $\begin{array}{l}\text { UE class } \\
\text { notation }\end{array}$ & $\begin{array}{c}\text { BS } \\
\text { coverage }\end{array}$ & $\begin{array}{l}\text { Potential } \\
\text { D2D UE }\end{array}$ & $\begin{array}{c}\text { D2D } \\
\text { truncation }\end{array}$ & $\begin{array}{c}\text { IP } \\
\text { satisfied }\end{array}$ & Mode & $\begin{array}{c}\text { Intensity for } \\
\text { Scheme } 1\end{array}$ & $\begin{array}{c}\text { Intensity for } \\
\text { Scheme } 2\end{array}$ \\
\hline Both & I. (c) & $x$ & $x$ & - & - & outage & $(\mathcal{U}-\mathcal{D}) \mathcal{O}_{p}$ & $(\mathcal{U}-\mathcal{D}) \mathcal{O}_{p}$ \\
\hline Both & II. (b) & $\checkmark$ & $x$ & - & - & cellular & $\left(1-\mathcal{O}_{p}\right)(\mathcal{U}-\mathcal{D})$ & $\left(1-\mathcal{O}_{p}\right)(\mathcal{U}-\mathcal{D})$ \\
\hline Both & III. (a) & $x$ & $\checkmark$ & $x$ & $\checkmark$ & $\mathrm{D} 2 \mathrm{D}$ & $\mathcal{O}_{p} \mathcal{U}_{d}$ & $\mathcal{O}_{p} \mathcal{D} \mathcal{P}_{d_{1}}$ \\
\hline Both & III. (c) & $x$ & $\checkmark$ & $x$ & $x$ & outage & $\mathcal{O}_{p}\left(1-\mathcal{P}_{d}\right) p \mathcal{D}$ & $\mathcal{O}_{p}\left(p-\mathcal{P}_{d_{1}}\right) \mathcal{D}$ \\
\hline Both & IV. (a) & $\checkmark$ & $\checkmark$ & $x$ & $\checkmark$ & $\mathrm{D} 2 \mathrm{D}$ & $\left(1-\mathcal{O}_{p}\right) \mathcal{U}_{d}$ & $\left(1-\mathcal{O}_{p}\right) \mathcal{D} \mathcal{P}_{d_{1}}$ \\
\hline Both & IV. (b) & $\checkmark$ & $\checkmark$ & $x$ & $x$ & cellular & $\left(1-\mathcal{O}_{p}\right)\left(1-\mathcal{P}_{d}\right) p \mathcal{D}$ & $\left(1-\mathcal{O}_{p}\right)\left(p-\mathcal{P}_{d_{1}}\right) \mathcal{D}$ \\
\hline 2 & V. (a) & $x$ & $\checkmark$ & $\checkmark$ & $\checkmark$ & $\mathrm{D} 2 \mathrm{D}$ & (- & $\mathcal{P}_{d_{0}} \mathcal{D}$ \\
\hline Both & V. (b) & $\checkmark$ & $\checkmark$ & $\checkmark$ & $\sqrt{ }$ or $x$ & cellular & $\left(1-\mathcal{O}_{p}\right)(1-p) \mathcal{D}$ & $\left(1-\mathcal{O}_{p}\right)(1-p) \mathcal{D}$ \\
\hline Both & V. (c) & $x$ & $\checkmark$ & $\checkmark$ & $x$ & outage & $(1-p) \mathcal{D O} \mathcal{O}_{p}$ & $\left(\mathcal{O}_{p}(1-p)-\mathcal{P}_{d_{2}}\right) \mathcal{D}$ \\
\hline
\end{tabular}

TABLE I: Classification of the different UEs in the two schemes.

control the extent to which the D2D communication is enabled in the network. An interpretation for the mode selection scheme is that the UEs select D2D mode if the power required to communicate in the D2D mode is less than the biased power required to communicate in the cellular mode. On one extreme, setting $T_{d}$ to zero disables D2D communication; on the other extreme, setting $T_{d}$ to $\infty$ enforces all potential D2D UEs to communicate in the D2D mode.

The UEs are classified into groups according to the distance between the transmit and receive UEs, the distance to the nearest BS, $T_{d}, \rho_{c}, \rho_{d}$, and the mode selection scheme. This classification is described in Table I. The two mode selection schemes are described in Table II; Scheme 1 does not include lines 13-14 while Scheme 2 includes all. The difference between Scheme 1 and 2 lies in that all potential D2D UEs that are in D2D truncation, go into outage in Scheme 1. In Scheme 2 , on the other hand, they go into outage only if they do not satisfy IP; however, if IP is satisfied, they transmit with the maximum power, $P_{u}$ (as the required power $r_{d}^{\eta_{d}} \rho_{d}$ is greater than $P_{u}$ due to D2D truncation). Hence, Scheme 2 reduces D2D-truncation outage. To summarize, UEs III. (a), IV. (a), and V. (a) (for Scheme 2) transmit in the D2D mode; UEs in II. (b), IV. (b), and V. (b) transmit in the cellular mode; while UEs in I. (c), III. (c), and V. (c) are in outage. Note, UEs that end with the letter: (a) transmit in the D2D mode, (b) transmit in the cellular mode, and (c) are in outage.

The intensities of the UEs in Scheme 1 and 2 are given in Table I. It ought to be noted that both mode selection schemes ensure an IP for the cellular UEs, and the IP value can be varied by varying $T_{d}$. Although this IP is not necessary in the overlay case, as the D2D transmitters do not operate on the same channel and therefore do not interfere with the cellular UEs, $T_{d}$ is included in the mode selection to control the traffic offloaded to the D2D mode and to control the D2D performance. Further, similar system models for the overlay and underlay cases guarantees a fair comparison.

The probability that a potential D2D UE selects the D2D mode of operation is given by $\mathcal{P}_{d}=\mathcal{P}_{d_{1}}+\mathcal{P}_{d_{2}}$, where $\mathcal{P}_{d_{1}}$ and $\mathcal{P}_{d_{2}}$ are the probability to select the D2D mode for, respectively, a potential D2D not in truncation and potential D2D in truncation. It can be shown that, $\mathcal{P}_{d_{1}}=\frac{\eta_{c}}{2 \eta_{d}}\left(\frac{T_{d} \rho_{c} b}{(\pi \lambda)^{\frac{\eta_{c}}{2}} P_{u}}\right)^{\frac{1}{\eta_{d}}} \gamma\left(\frac{\eta_{c}}{2 \eta_{d}}, \pi \lambda\left(\frac{P_{u}}{\rho_{c} T_{d}}\right)^{\frac{2}{\eta_{c}}}\right)$; where $\gamma(a, b)=\int_{0}^{b} x^{a-1} e^{-x} d x, b=1$ for Scheme 1 , and $b=\rho_{\min } / \rho_{d}$ for Scheme 2. For Scheme 1, $\mathcal{P}_{d_{2}}=$ 0 ; for Scheme $2, \mathcal{P}_{d_{2}}=\min \left((1-p) \mathcal{O}_{p}, \mathcal{P}_{d_{3}}\right)$, where $\mathcal{P}_{d_{3}}=\frac{\eta_{c}}{2 \eta_{d}}\left(\frac{T_{d} \rho_{c} \rho_{\min }}{(\pi \lambda)^{\frac{\eta_{c}}{2}} P_{u} \rho_{d}}\right)^{\frac{1}{\eta_{d}}}\left[\gamma\left(\frac{\eta_{c}}{2 \eta_{d}}, \pi \lambda\left(\frac{P_{u} \rho_{d}}{\rho_{c} \rho_{\min } T_{d}}\right)^{\frac{2}{\eta_{c}}}\right)-\right.$ $\left.\gamma\left(\frac{\eta_{c}}{2 \eta_{d}}, \pi \lambda\left(\frac{P_{u}}{\rho_{c} T_{d}}\right)^{\frac{2}{\eta_{c}}}\right)\right]$. The intensity of the D2D links for Scheme 1 is given by $\mathcal{U}_{d}=p \mathcal{D} \mathcal{P}_{d}$ and for Scheme 2 by $\mathcal{U}_{d}=\mathcal{D} \mathcal{P}_{d}$.

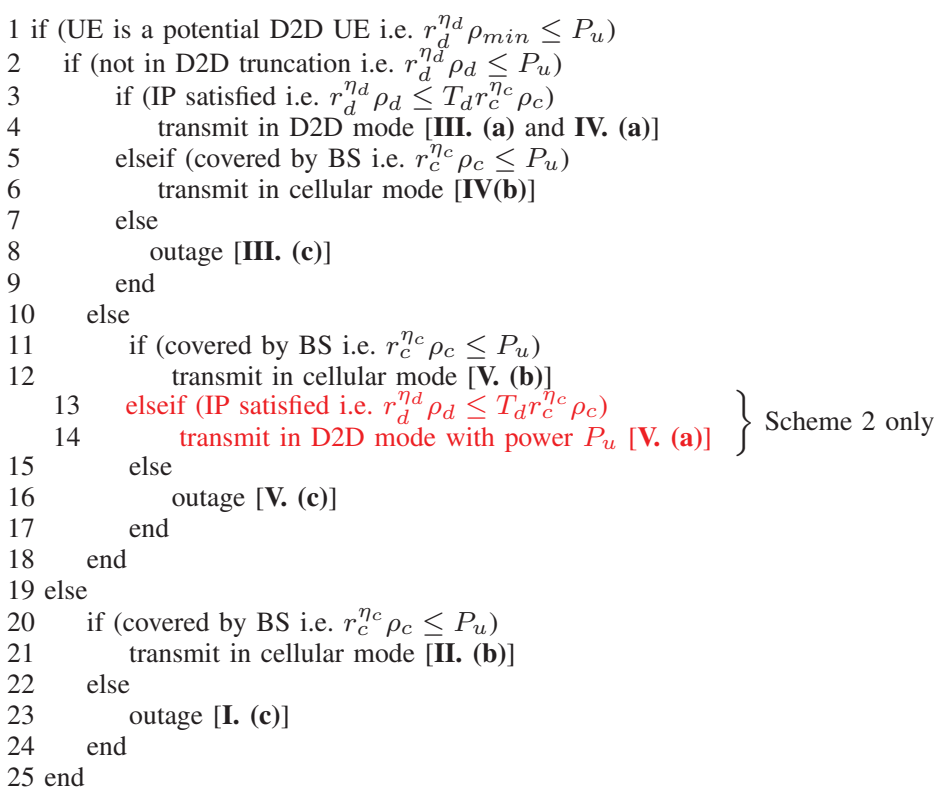

TABLE II: Mode selection criteria for the two schemes. Scheme 1 does not include the lines highlighted in red, while Scheme 2 includes all the lines.

\section{AnAlysis of Transmit Powers AND SINR}

\section{A. Statistics of Transmit Powers}

In this subsection, we characterize the transmit powers using their PDF's and $\alpha^{t h}$ moments (for $\alpha>0$ ). These statistics hold for both underlay and overlay. Due to the page limit, we have not given the proofs, but our results can be re-derived by following the footsteps of the work in [12], using our mode selection schemes and D2D link distance distribution.

For both Schemes 1 and 2, UEs that can be classified into III. (a) or IV. (a), operating in the D2D mode, have transmit 
power $P_{3 a 4 a}=\left\{\rho_{d} r_{d}^{\eta_{d}}: \rho_{d} r_{d}^{\eta_{d}} \leq T_{d} \rho_{c} r_{c}^{\eta_{c}}\right\}$. The PDF is given by,

$$
f_{P_{3 a 4 a}}(x)=\frac{2 x^{\frac{1}{\eta_{d}}-1} e^{-\pi \lambda\left(\frac{x}{\rho_{c} T_{d}}\right)^{\frac{2}{\eta_{c}}}}(\pi \lambda)^{\frac{\eta_{c}}{2 \eta_{d}}}}{\eta_{c}\left(\rho_{c} T_{d}\right)^{\frac{1}{\eta_{d}}} \gamma\left(\frac{\eta_{c}}{2 \eta_{d}}, \pi \lambda\left(\frac{P_{u}}{\rho_{c} T_{d}}\right)^{\frac{2}{\eta_{c}}}\right)},
$$

for $0 \leq x \leq P_{u}$ and the $\alpha^{t h}$ moment of $P_{3 a 4 a}$ by,

$$
\mathbb{E}\left[P_{3 a 4 a}^{\alpha}\right]=\frac{\left(T_{d} \rho_{c}\right)^{\alpha} \gamma\left(\frac{\alpha \eta_{c}}{2}+\frac{\eta_{c}}{2 \eta_{d}}, \pi \lambda\left(\frac{P_{u}}{\rho_{c} T_{d}}\right)^{\frac{2}{\eta_{c}}}\right)}{(\pi \lambda)^{\frac{\alpha \eta_{c}}{2}} \gamma\left(\frac{\eta_{c}}{2 \eta_{d}}, \pi \lambda\left(\frac{P_{u}}{\rho_{c} T_{d}}\right)^{\frac{2}{\eta_{c}}}\right)} .
$$

UEs of Scheme 2, operating in the D2D mode, that are classified as UEs in V. (a) have, $f_{P_{5 a}}(x)=1$ at $x=P_{u}$, and 0 otherwise; hence $\mathbb{E}\left[P_{5 a}^{\alpha}\right]=P_{u}^{\alpha}$. Since the average D2D power is just a weighted sum of when the D2D mode occurs, for Scheme $1, \mathbb{E}\left[P_{d}^{\alpha}\right]=\mathbb{E}\left[P_{3 a 4 a}^{\alpha}\right]$, while for Scheme 2, $\mathbb{E}\left[P_{d}^{\alpha}\right]=\frac{\mathcal{P}_{d_{1}}}{\mathcal{P}_{d}} \mathbb{E}\left[P_{3 a 4 a}^{\alpha}\right]+\frac{\mathcal{P}_{d_{2}}}{\mathcal{P}_{d}} P_{u}^{\alpha}$.

In the case of the cellular users, we first consider the UEs in II. (b). For both schemes, the transmit power of these UEs is $P_{2 b}=\left\{\rho_{c} r_{c}^{\eta_{c}}: \rho_{c} r_{c}^{\eta_{c}}<P_{u}\right\}$, has PDF

$$
f_{P_{2 b}}(x)=\frac{2 \pi \lambda x^{\frac{2}{\eta_{c}}-1} e^{-\pi \lambda\left(\frac{x}{\rho_{c}}\right) \frac{2}{\eta_{c}}}}{\eta_{c} \rho_{c}^{\frac{2}{\eta_{c}}}\left(1-e^{-\pi \lambda\left(\frac{P_{u}}{\rho_{c}}\right)^{\frac{2}{\eta_{c}}}}\right)} \quad 0 \leq x \leq P_{u}
$$

and the $\alpha^{t h}$ moment of the transmit power is given by,

$$
\mathbb{E}\left[P_{2 b}^{\alpha}\right]=\frac{\rho_{c}^{\alpha} \gamma\left(\frac{\alpha \eta_{c}}{2}+1, \pi \lambda\left(\frac{P_{u}}{\rho_{c}}\right)^{\frac{2}{\eta_{c}}}\right)}{(\pi \lambda)^{\frac{\alpha \eta_{c}}{2}}\left(1-e^{-\pi \lambda\left(\frac{P_{u}}{\rho_{c}}\right)^{\frac{2}{\eta_{c}}}}\right)} .
$$

The other UEs operating in cellular mode are those in IV. (b). For both Schemes 1 and 2, these UEs transmit with power $P_{4 b}=\left\{\rho_{c} r_{c}^{\eta_{c}}: \rho_{c} r_{c}^{\eta_{c}}<P_{u} \cap \rho_{c} r_{c}^{\eta_{c}} \leq \frac{1}{T_{d}} \rho_{d} r_{d}^{\eta_{d}}\right\}$. The PDF of this power is given by,

$$
f_{P_{4 b}}(x)=\frac{2 \pi \lambda x^{\frac{2}{\eta_{c}}-1} e^{-\pi \lambda\left(\frac{x}{\rho_{c}}\right)^{\frac{2}{\eta_{c}}}}\left(1-\left(\frac{x T_{d}}{P_{u}}\right)^{\frac{1}{\eta_{d}}}\right)}{\eta_{c} \rho_{c}^{\frac{2}{\eta_{c}}}\left(1-e^{-\pi \lambda\left(\frac{P_{u}}{\rho_{c}}\right)}\right)},
$$

for $0 \leq x \leq \frac{P_{u}}{\max \left(T_{d}, 1\right)}$; where,

$$
\begin{aligned}
& \left.\mathbb{P}\left(\widetilde{X}_{c} \leq \frac{X_{d}}{T_{d}}\right)=\left(1-\min \left(1, T_{d}^{\frac{1}{\eta_{d}}}\right)\right)+\frac{1}{\left(1-e^{-\pi \lambda\left(\frac{P_{u}}{\rho_{c}}\right)} \frac{2}{\eta_{c}}\right.}\right) \times \\
& {\left[\min \left(1, T_{d}^{\frac{1}{\eta_{d}}}\right)-\left(\frac{T_{d} \rho_{c}}{P_{u}}\right)^{\frac{1}{\eta_{d}}} \frac{\eta_{c}}{2 \eta_{d}(\pi \lambda)^{\frac{\eta_{c}}{2 \eta_{d}}}} \gamma\left(\frac{\eta_{c}}{2 \eta_{d}}, \pi \lambda\left(\frac{P_{u}}{\rho_{c}}\right)^{\frac{2}{\eta_{c}}}\right)\right] \cdot=1-e^{-\frac{\theta}{\rho_{\chi}}\left(\sigma^{2}+\mathcal{I}_{c \chi}+\mathcal{I}_{d \chi}\right)}=1-e^{-\frac{\theta}{\rho_{\chi}} \sigma^{2}} \mathcal{L}_{\mathcal{I}_{c \chi}}\left(\frac{\theta}{\rho_{\chi}}\right) \mathcal{L}_{\mathcal{I}_{d \chi}}\left(\frac{\theta}{\rho_{\chi}}\right),}
\end{aligned}
$$

The $\alpha^{t h}$ moment of the power $P_{4 b}$ is given by,

$$
\begin{aligned}
& \mathbb{E}\left[P_{4 b}^{\alpha}\right]=\frac{1}{\mathbb{P}\left(\widetilde{X}_{c} \leq \frac{X_{d}}{T_{d}}\right)}\left[\frac{\rho_{c}^{\alpha} \gamma\left(\frac{\alpha \eta_{c}}{2}+1, \pi \lambda\left(\frac{P_{u}}{\max \left(1, T_{d}\right) \rho_{c}}\right)^{\frac{2}{\eta_{c}}}\right)}{(\pi \lambda)^{\frac{\alpha \eta_{c}}{2}}\left(1-e^{-\pi \lambda\left(\frac{P_{u}}{\rho_{c}}\right)^{\frac{2}{\eta_{c}}}}\right)}-\right. \\
& \left.\frac{T_{d}^{\frac{1}{\eta_{d}}} \rho_{c}^{\alpha+\frac{1}{\eta_{d}}} \gamma\left(\frac{\eta_{c}}{2 \eta_{d}}+\frac{\alpha \eta_{c}}{2}+1, \pi \lambda\left(\frac{P_{u}}{\max \left(1, T_{d}\right) \rho_{c}}\right)^{\frac{2}{\eta_{c}}}\right)}{(\pi \lambda)^{\frac{\eta_{c}}{2 \eta_{d}}+\frac{\alpha \eta_{c}}{2}} P_{u}^{\frac{1}{\eta_{d}}}\left(1-e^{-\pi \lambda\left(\frac{P_{u}}{\rho_{c}}\right)^{\frac{2}{\eta_{c}}}}\right)}\right] .
\end{aligned}
$$

The transmit power of the UEs which are in V. (b) is $P_{5 b}=\left\{\rho_{c} r_{c}^{\eta_{c}}: \rho_{c} r_{c}^{\eta_{c}}<P_{u}\right\}$, which is equivalent to the case of the UEs in II. (b). Hence, $f_{P_{5 b}}(x)=f_{P_{2 b}}(x)$ and $\mathbb{E}\left[P_{5 b}^{\alpha}\right]=\mathbb{E}\left[P_{2 b}^{\alpha}\right]$. Additionally, the $\alpha^{t h}$ moment of a UE operating in the cellular mode is a weighted sum of the the different powers of UEs in the cellular mode. For Scheme $1, \mathbb{E}\left[P_{c}^{\alpha}\right]=\left(\frac{\mathcal{U}-p \mathcal{D}}{\mathcal{U}-\mathcal{U}_{d}}\right) \mathbb{E}\left[P_{2 b}^{\alpha}\right]+\left(\frac{\left(1-\mathcal{P}_{d}\right) p \mathcal{D}}{\mathcal{U}-\mathcal{U}_{d}}\right) \mathbb{E}\left[P_{4 b}^{\alpha}\right]$; while for Scheme 2, $\mathbb{E}\left[P_{c}^{\alpha}\right]=\left(\frac{\mathcal{U}-p \mathcal{D}}{\mathcal{U}-\mathcal{P}_{d_{1}} \mathcal{D}}\right) \mathbb{E}\left[P_{2 b}^{\alpha}\right]+\left(\frac{\left(p-\mathcal{P}_{d_{1}}\right) \mathcal{D}}{\mathcal{U}-\mathcal{P}_{d_{1}} \mathcal{D}}\right) \mathbb{E}\left[P_{4 b}^{\alpha}\right]$.

\section{B. SINR Analysis}

We represent by $\widetilde{\Phi}_{c}$ and $\widetilde{\Phi}_{d}$ the UEs operating in the cellular and D2D modes respectively. Neither of these is a PPP and they are not independent as they depend on the same set of BSs. However, for analytical tractability, we assume that $\widetilde{\Phi}_{c}$ and $\widetilde{\Phi}_{d}$ are independent PPP. Our assumption is a good estimate as the density of the PPPs and the interference exclusion region around the test receiver has been estimated carefully. The PPP assumption is validated in the next section. The intensity of $\widetilde{\Phi}_{c}$ used is $\lambda$ because we have assumed saturation conditions (i.e. $\mathcal{U} \gg \lambda$ ) and the intensity of $\widetilde{\Phi}_{d}$ is the intensity of the D2D links, $\mathcal{U}_{d}$. The exclusion region for cellular receivers has been calculated using the IP boundary; the D2D receivers, on the other hand, lack IP protection. It is important to note that the PPP assumption only ignores the mutual correlations between the positions of simultaneously active UEs, as the IP condition captures the correlation between the interfering UEs and the test transmitter and receiver.

We have characterized the SINR by its cumulative distribution function (CDF). A UE operates in mode $\chi \in\{c, d\}$, where $c$ and $d$ denote the cellular and D2D modes respectively. For Scheme 1, in the underlay case, we define the SINR of the users in mode $\chi$ as follows; $S I N R_{\chi}=\frac{\rho_{\chi} h_{0}}{\sigma^{2}+\mathcal{I}_{c \chi}+\mathcal{I}_{d \chi}}$, where $\mathcal{I}_{\kappa \chi}=\sum_{u_{i} \in \widetilde{\Phi}_{\kappa}} P_{\kappa_{i}} h_{i}\left\|y-u_{i}\right\|^{-\eta_{\chi}}$. The interference of the cellular users (D2D users) to the UE being considered in mode $\chi$ is denoted by $\mathcal{I}_{c \chi}\left(\mathcal{I}_{d \chi}\right)$, and $\sigma^{2}$ denotes the noise power. Since in the overlay case cross-mode interference does not exist, the expression for the SINR simplifies to $S I N R_{\chi}=\frac{\rho_{\chi} h_{0}}{\sigma^{2}+\mathcal{I}_{\chi \chi}}$. 
where $\mathcal{L}_{X}$ is the Laplace transform of the PDF of the RV $X$. Similarly, in the overlay case the CDF of the SINR is,

$$
\mathbb{P}\left(S I N R_{\chi} \leq \theta\right)=1-\exp \left(-\frac{\theta}{\rho_{\chi}} \sigma^{2}\right) \mathcal{L}_{\mathcal{I}_{\chi \chi}}\left(\frac{\theta}{\rho_{\chi}}\right)
$$

The Laplace transforms of the interference's are,

$$
\begin{aligned}
& \mathcal{L}_{\mathcal{I}_{d d}}=\exp \left(-\pi \mathcal{U}_{d} s^{\frac{2}{\eta_{d}}} \mathbb{E}\left[P_{d}^{\frac{2}{\eta_{d}}}\right] \Gamma\left(1+\frac{2}{\eta_{d}}\right) \Gamma\left(1-\frac{2}{\eta_{d}}\right)\right) \\
& \mathcal{L}_{\mathcal{I}_{c d}}=\exp \left(-\pi \lambda s^{\frac{2}{\eta_{d}}} \mathbb{E}\left[P_{c}^{\frac{2}{\eta_{d}}}\right] \Gamma\left(1+\frac{2}{\eta_{d}}\right) \Gamma\left(1-\frac{2}{\eta_{d}}\right)\right) \\
& \mathcal{L}_{\mathcal{I}_{c c}}=\exp \left(-2 \pi \lambda s^{\frac{2}{\eta_{c}}} \mathbb{E}\left[P_{c}^{\frac{2}{\eta_{c}}}\right] \int_{\left.\left(\frac{1}{s \rho_{c}}\right)^{\frac{1}{\eta_{c}}}\left(\frac{y}{y^{\eta_{c}}+1}\right) d y\right)}\right. \\
& \stackrel{\eta_{c}=4}{=} \exp \left(-\pi \lambda \sqrt{s} \mathbb{E}\left[\sqrt{P_{c}}\right] \arctan \left(\sqrt{s \rho_{c}}\right)\right) \\
& \stackrel{\mathcal{L}_{\mathcal{I}_{d c}}}{=}=\exp \left(-2 \pi \mathcal{U}_{d} s^{\frac{2}{\eta_{c}}} \mathbb{E}\left[P_{d}^{\frac{2}{\eta_{c}}}\right]\left(\frac{1}{s \rho_{c} T_{d}}\right)^{\frac{1}{\eta_{c}}}\left(\frac{y}{y^{\eta_{c}}+1}\right) d y\right) \\
& \stackrel{\eta_{c}=4}{=} \exp \left(-\pi \mathcal{U}_{d} \sqrt{s} \mathbb{E}\left[\sqrt{P_{d}}\right] \arctan \left(\sqrt{s \rho_{c} T_{d}}\right)\right)
\end{aligned}
$$

Additionally, for Scheme 1, the link spectrum efficiency for a UE operating in mode $\chi$ is given as follows, where $\xi=\frac{\left(e^{t}-1\right)}{\rho_{\chi}}$ :

$$
\begin{aligned}
& \mathcal{R}_{\chi}=\mathbb{E}\left[\ln \left(1+S I N R_{\chi}\right)\right] \\
& =\int_{0}^{\infty} \mathbb{P}\left(\ln \left(1+S I N R_{\chi}\right)>t\right) d t \\
& =\int_{0}^{\infty} \exp \left(-\xi \sigma^{2}\right) \mathcal{L}_{\mathcal{I}_{c \chi}}(\xi) \mathcal{L}_{\mathcal{I}_{d \chi}}(\xi) d t \quad \text { (for underlay) } \\
& =\int_{0}^{\infty} \exp \left(-\xi \sigma^{2}\right) \mathcal{L}_{\mathcal{I}_{\chi \chi}}(\xi) d t \quad \text { (for overlay). }
\end{aligned}
$$

$S I N R_{d}$ in Scheme 2 is different from that in Scheme 1, as the UEs operating in $\mathbf{V}$. (a) do not achieve $\rho_{d}$ at their receiver. Hence, the D2D SINR is a weighted sum of the SINR of the UEs in III. (a), IV. (a) and V. (a). In the underlay case, it is given by $S I N R_{d}=\frac{\mathcal{D P}_{d_{1}} \rho_{d} h_{0}}{\mathcal{U}_{d}\left(\sigma^{2}+\mathcal{I}_{c d}+\mathcal{I}_{d d}\right)}+\frac{\mathcal{D P} \mathcal{P}_{d_{2}} P_{u} r_{d}^{-\eta} h_{0}}{\mathcal{U}_{d}\left(\sigma^{2}+\mathcal{I}_{c d}+\mathcal{I}_{d d}\right)}$. The overlay case simply does not include the $\mathcal{I}_{c d}$ terms.

Due to this, for the case of Scheme 2, the complementary $\mathrm{CDF}(\mathrm{CCDF})$ of the D2D SINR for underlay is,

$$
\begin{aligned}
& \mathbb{P}\left(S I N R_{d}>\theta\right)=\frac{\mathcal{D} \mathcal{P}_{d_{1}}}{\mathcal{U}_{d}}\left(e^{-\frac{\theta}{\rho_{d}} \sigma^{2}} \mathcal{L}_{\mathcal{I}_{c d}}\left(\frac{\theta}{\rho_{d}}\right) \mathcal{L}_{\mathcal{I}_{d d}}\left(\frac{\theta}{\rho_{d}}\right)\right) \\
& +\frac{\mathcal{D} \mathcal{P}_{d_{2}}}{\mathcal{U}_{d}}\left(\int_{R}^{R_{\max }} \frac{e^{-\frac{\theta x \eta_{d}}{P_{u}} \sigma^{2}}}{R_{\max }} \mathcal{L}_{\mathcal{I}_{c d}}\left(\frac{\theta x^{\eta_{d}}}{P_{u}}\right) \mathcal{L}_{\mathcal{I}_{d d}}\left(\frac{\theta x^{\eta_{d}}}{P_{u}}\right) d x\right.
\end{aligned}
$$

The CCDF for the overlay case does not include the $\mathcal{L}_{\mathcal{I}_{c d}}$ terms.
Also, for Scheme 2, the D2D link spectrum efficiency in the underlay case, where $\xi=\frac{\left(e^{t}-1\right)}{\rho_{d}}$, is:

$$
\begin{aligned}
& \mathcal{R}_{d}=\frac{\mathcal{D} \mathcal{P}_{d_{1}}}{\mathcal{U}_{d}} \int_{0}^{\infty} e^{-\xi \sigma^{2}} \mathcal{L}_{\mathcal{I}_{c d}}(\xi) \mathcal{L}_{\mathcal{I}_{d d}}(\xi) d t \\
& +\frac{\mathcal{D} \mathcal{P}_{d_{2}}}{\mathcal{U}_{d}} \int_{0}^{\infty} \int_{R}^{R_{\max }} \frac{e^{-\frac{\xi \rho_{d} x_{d}}{P_{u}} \sigma^{2}}}{R_{\max }} \mathcal{L}_{\mathcal{I}_{c d}}\left(\frac{\xi \rho_{d} x^{\eta_{d}}}{P_{u}}\right) \mathcal{L}_{\mathcal{I}_{d d}}\left(\frac{\xi \rho_{d} x^{\eta_{d}}}{P_{u}}\right) d x d t .
\end{aligned}
$$

Again, the overlay case does not include the $\mathcal{L}_{\mathcal{I}_{c d}}$ terms. Note: the cellular UEs have the same SINR, and therefore SINR CDF and link spectrum efficiency for both Schemes 1 and 2 .

Assuming a fair (i.e. equal) resource allocation in the BSs, the average share that a generic cellular user gets is given by $\beta=\frac{\text { BS intensity }}{\text { intensity of UEs in cellular mode }}$, therefore for Scheme $1, \beta=$ $\frac{\lambda}{\left(1-\mathcal{O}_{p}\right)\left(\mathcal{U}-\mathcal{U}_{d}\right)}$, while for Scheme $2, \beta=\frac{\lambda}{\left(1-\mathcal{O}_{p}\right)\left(\mathcal{U}-\mathcal{D} \mathcal{P}_{d_{1}}\right)}$. Two metrics other than outage probability are used for analyzing performance in this work: the average spectral efficiency per potential D2D UE, $\mathcal{R}_{a v g}=\mathcal{P}_{d} \mathcal{R}_{d}+\frac{\beta}{2}\left(1-\mathcal{O}_{p}\right)\left(1-\mathcal{P}_{d_{1}}\right) \mathcal{R}_{c}$, and the spatial spectral efficiency from the network's perspective, $\mathcal{T}_{n}=\mathcal{U}_{d} \mathcal{R}_{d}+\lambda \mathcal{R}_{c}$. Note that for measuring $\mathcal{R}_{\text {avg }}$, the spectral efficiency of the cellular link is multiplied by 0.5 to reflect two hop nature of the cellular link (i.e. uplink then downlink). Also, it is natural to estimate the cellular link rate by the uplink rate as the uplink is usually the bottleneck link.

\section{RESUltS AND ANALYSIS}

Unless stated otherwise, the BS intensity is $\lambda=10 \mathrm{BS} / \mathrm{km}^{2}$, the intensity of the UEs is $\mathcal{U}=100 \mathrm{UE} / \mathrm{km}^{2}$, the potential D2D UEs intensity is $\mathcal{D}=50 \mathrm{UE} / \mathrm{km}^{2}$, the maximum transmit power is $P_{u}=200 \mathrm{~mW}$, the receiver sensitivity is $\rho_{\min }=-90$ $\mathrm{dBm}$, the cutoff threshold for cellular UEs is $\rho_{c}=-80 \mathrm{dBm}$, the cutoff threshold for D2D UEs is $\rho_{d}=\frac{\rho_{c}}{r}$, the value of $r$ is 1 , the $\mathrm{D} 2 \mathrm{D}$ bias factor $T_{d}=1$, the path-loss exponents are $\eta_{c}=\eta_{d}=4$, the SINR threshold $\theta=1$, and the noise power is $\sigma^{2}=-90 \mathrm{dBm}$. Note: in this work, altering $\rho_{c}$ alters $\rho_{d}$ (due to its definition), while altering $r$ alters $\rho_{d}$ but not $\rho_{c}$.

\section{A. Underlay}

Increasing $\rho_{c}$ (and therefore $\rho_{d}$ ) causes a higher SINR requirement at the receivers and therefore causes an increase in truncation outage for both cellular and D2D UEs. However, the users that do transmit have better SINR due to the increased signal component as well as lower interference due to the fewer interferers. This results in SINR outage decreasing with increasing $\rho_{c}$, for a constant $r$ and $T_{d}$, as shown in Fig. 1 for Scheme 2. Increasing $T_{d}$ enables more D2D communication, thus causing greater interference from D2D UEs, which deteriorates the SINR for both communication modes, resulting in higher outage; this is shown, for Scheme 2 in Fig. 2. Scheme 1 has the same trend for increasing $\rho_{c}$ and $T_{d}$ as Scheme 2; these have not been plotted for brevity and can be found in [12]. Increasing $r$ decreases $\rho_{d}$ ( $\rho_{c}$ remains constant), which reduces the D2D truncation probability and therefore increases the D2D interferers in the system resulting in SINR deterioration of both cellular and D2D UEs. It should be noted that the impact on the SINR of D2D UEs is larger than that on cellular 

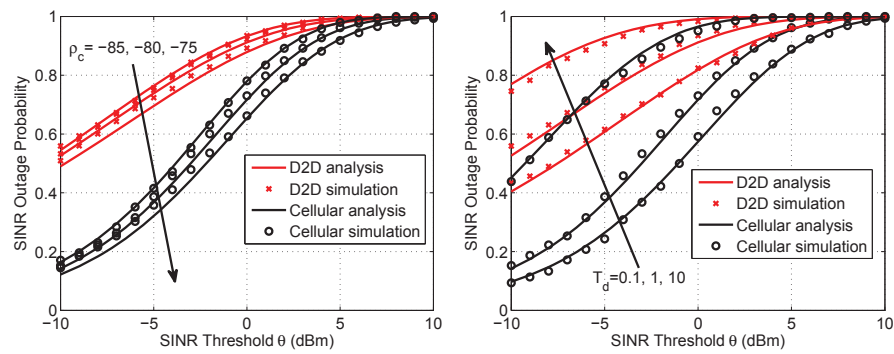

Fig. 1: Scheme 2: SINR outage vs. $\theta$ for $r=1, T_{d}=1$ and different $\rho_{c}$.

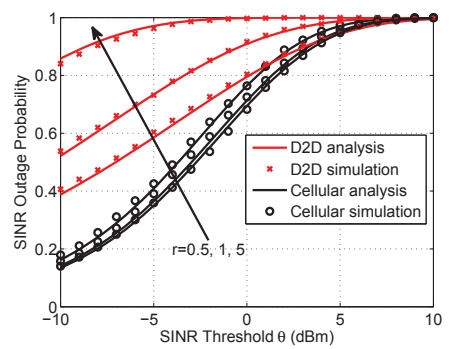

Fig. 3: Scheme 1: SINR outage vs. $\theta$ for $\rho_{c}=-80 \mathrm{dBm}$, $T_{d}=1$ and different $r$.

Fig. 2: Scheme 2: SINR outage vs. $\theta$ for $\rho_{c}=-80 \mathrm{dBm}$, $r=1$ and different $T_{d}$.

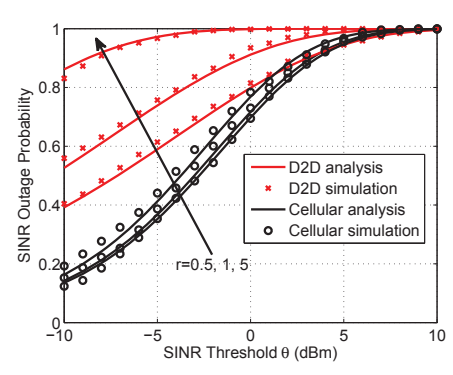

Fig. 4: Scheme 2: SINR outage vs. $\theta$ for $\rho_{c}=-80 \mathrm{dBm}$, $T_{d}=1$ and different $r$.

UEs which only have to deal with higher interference; the D2D users, additionally, have lower signal components due to the reduced threshold $\rho_{d}$. Hence increasing $r$ deteriorates SINR outage of both cellular and D2D UEs, impacting the D2D UEs more as shown in Figs. 3 and 4 for the two schemes. The results in Figs. 1 - 4 validate our analysis by showing that the models being used accurately capture the SINR outage.

Increasing $r$ causes the SINR of both D2D and cellular UEs to deteriorate leading to lower rates, as reflected by the decrease in $\mathcal{R}_{\text {avg }}$ and $\mathcal{T}_{n}$ in Figs. 5 and 6. Moreover, the optimal $T_{d}$ for the spectral efficiencies decreases with increasing $r$. As higher $r$ leads to worse D2D-SINR than cellular-SINR, the D2D mode performs more poorly than the cellular; since $T_{d}$ enhances the use of the D2D mode, the optimal $T_{d}$ decreases with increasing $r$ due to the lower-quality D2D mode. This is in contrast to the case of increasing $\rho_{c}$ (and $\rho_{d}$ ), where the optimal $T_{d}$ remains constant because the cellular and D2D modes are both impacted in the same way and hence perform similarly [12].

In general, $\mathcal{R}_{\text {avg }}$ and $\mathcal{T}_{n}$ have similar trends; with increasing $r$, the value of the spectral efficiencies first increases and then decreases. This is because below a certain optimal value, increasing $r$, which increases spatial frequency reuse, improves the SINR and therefore the spectral efficiencies, by increasing the number of contributing UEs. After the optimal, however, the effect of the increased D2D interferers in the system dominates the benefit of the larger number of active users, resulting in deteriorating SINR and spectral efficiency. Additionally, at higher $r$, the signal part of the contributing
D2D UEs is poor too further worsening D2D SINR. This can be seen in Figs. 7 - 10. It ought to be mentioned that the range of $r$ being considered in Figs. 7 - 10 is low; this has been done to observe the optimal values of $r$. Note, the optimal is not visible in Figs. 7 and 9, for Scheme 2, because they are being masked by the higher values of Scheme 1; and for Scheme 1, because the optimal occurs at very low values of $r$ that are not in the range plotted. Figs. 7 and 8 show spectral efficiencies decrease with decreasing $\rho_{c}$ (and $\rho_{d}$ ) as the SINR deteriorates. In general, increasing $T_{d}$ causes SINR, and therefore spectral efficiency, deterioration. However, Figs. 9 and 10 show spectral efficiencies increase with increasing $T_{d}$; this is because of the range of low $r$ being considered which has very few D2D interferers; increasing $T_{d}$ here leads to higher SINR and spectral efficiency. At higher $r$, however, there are many D2D interferers and increasing $T_{d}$ results in SINR and spectral efficiencies deteriorating.

An important observation from the results is that the overall network performance, in terms of outage probability and $\mathcal{T}_{n}$, is not significantly different for the two schemes; however, in terms of $\mathcal{R}_{\text {avg }}$ Scheme 1 significantly outperforms Scheme 2. This occurs because Scheme 2, by allowing more D2D UEs to communicate, reduces the average quality of a link. The SINR outage probability and $\mathcal{T}_{n}$, however, measure the overall network performance which is averaged over the total number of users. Since, the number of users in Scheme 2 is larger, the contribution to the SINR is larger, resulting in overall network performance similar to that of Scheme 1's. In other words, Scheme 2 caters to a larger number of users focusing less on the link quality, while Scheme 1 caters to fewer users providing better quality.

Optimal values exist that maximize spatial spectrum efficiencies for both $T_{d}$ and $r$ as they both impact the number of D2D UEs that can transmit. An observation from the results is that $r$ is a more robust criteria for flexible mode selection, in terms of both outage and spectral efficiency, as it impacts cellular outage significantly less than $T_{d}$ does and as the roll off of spectral efficiency after the optimal is much smoother in the case of $r$. This can be explained by noting that increasing $T_{d}$ makes the IP condition easier to satisfy and thereby increases $\mathcal{P}_{d}$, whereas increasing $r$ not only makes the IP condition easier to satisfy, but also decreases D2D truncation outage which remains unaffected in the case of varying $T_{d}$.

\section{B. Overlay}

Since the bandwidth is split between the two communication modes in the overlay case, we redefine the performance metrics as $\mathcal{R}_{\text {avg }}=\tau \mathcal{P}_{d} \mathcal{R}_{d}+(1-\tau) \frac{\beta\left(1-\mathcal{O}_{p}\right)\left(1-\mathcal{P}_{d_{1}}\right)}{2} \mathcal{R}_{c}$ and $\mathcal{T}_{n}=\tau \mathcal{U}_{d} \mathcal{R}_{d}+(1-\tau) \lambda \mathcal{R}_{c}$. Maximizing these metrics w.r.t. $\tau$ is in the form of the following optimization problem:

$$
\tau^{*}=\arg \max _{\tau \in[0,1]} \tau a \mathcal{R}_{d}+(1-\tau) b \mathcal{R}_{c},
$$

where $a$ and $b$ are constants that vary with the metric being maximized. From this we can see that the solution to the 

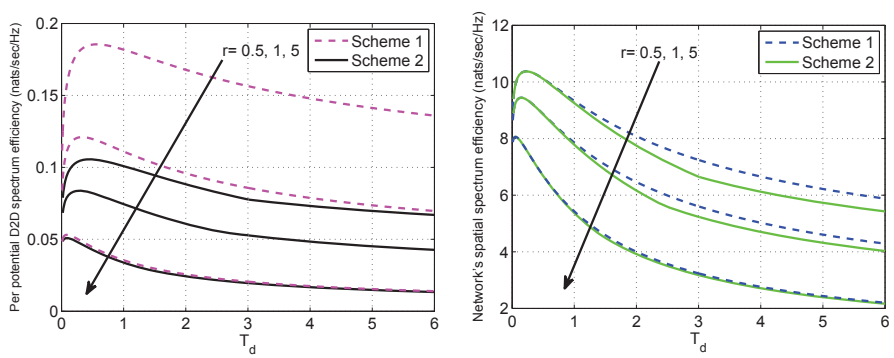

Fig. 5: $\mathcal{R}_{a v g}$ vs. $T_{d}$ for $\rho_{c}=$ $-80 \mathrm{dBm}$ and different $r$.

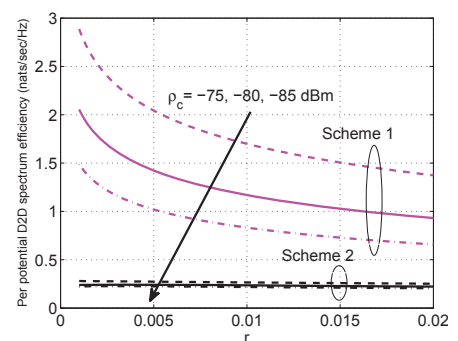

Fig. 7: $\mathcal{R}_{\text {avg }}$ vs. $r$ for $T_{d}=1$ and different $\rho_{c}$.

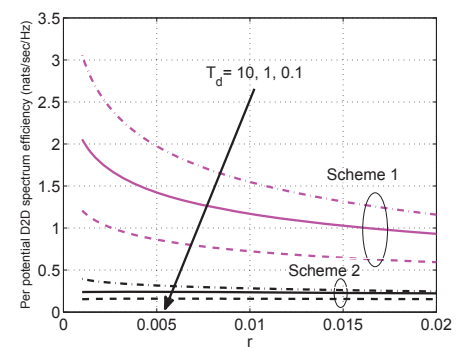

Fig. 9: $\mathcal{R}_{\text {avg }}$ vs. $r$ for $\rho_{c}=$ $-80 \mathrm{dBm}$ and different $T_{d}$.

Fig. 10: $\mathcal{T}_{n}$ vs. $r$ for $\rho_{c}=$ $-80 \mathrm{dBm}$ and different $T_{d}$.

optimization problem is simply: if $a \mathcal{R}_{d}>b \mathcal{R}_{c}, \tau^{*}=0$; otherwise, $\tau^{*}=1$. In other words, the optimal overlay criteria which maximizes spectral efficiency is to decide which mode performs better and assign all bandwidth to it. Since an optimal $\tau$ value, which is not 0 or 1 , does not exist based on our criteria, it suffices to say that optimal communication, in terms of maximizing spectral efficiency, in the overlay case makes us lose the essence of D2D communication.

\section{Conclusion}

We have presented and analyzed a comprehensive tractable framework for D2D-enabled cellular networks using two flexible mode selection schemes. The flexibility comes from the biasing factor $T_{d}$, which controls the amount of traffic offloaded to the D2D mode and the IP to cellular UEs from D2D UEs. The power-control cutoff thresholds required at the D2D receivers and BSs have been segregated in this work. The ratio $r$ of the cellular and D2D power-control cutoff thresholds also controls the amount of traffic offloaded to the D2D mode as well as the IP of the cellular UEs. We find that, like $T_{d}$, an optimal $r$ exists that maximizes spectral efficiency for D2D enabled networks, and $r$ in fact happens to be a more robust parameter to use when maximizing spectral efficiency. Additionally, our results show that Scheme 2, which prioritizes spatial frequency reuse over the per-user achievable performance compared to Scheme 1, attains almost the same overall network performance in terms of the SINR outage and network spectral efficiency. Hence, Scheme 2 trades the link quality to serve a larger quantity of users, maintaining almost the same overall network performance as Scheme 1.

\section{REFERENCES}

[1] A. Asadi, Q. Wang, and V. Mancuso, "A survey on device-to-device communication in cellular networks," IEEE Communications Surveys and Tutorials, vol. 16, no. 4, pp. 1801-1819, 2014.

[2] J. Andrews, F. Baccelli, and R. Ganti, "A tractable approach to coverage and rate in cellular networks," IEEE Trans. Commun., vol. 59, no. 11, pp. 3122-3134, Nov. 2011.

[3] H. ElSawy, E. Hossain, and M. Haenggi, "Stochastic geometry for modeling, analysis, and design of multi-tier and cognitive cellular wireless networks: A survey," IEEE Communications Surveys and Tutorials, vol. 15, no. 3, pp. 996-1019, Jul. 2013.

[4] A. Guo and M. Haenggi, "Spatial stochastic models and metrics for the structure of base stations in cellular networks," IEEE Trans. Wireless Commun., vol. 12, no. 11, pp. 5800-5812, Nov. 2013.

[5] H. Dhillon, R. Ganti, F. Baccelli, and J. Andrews, "Modeling and analysis of K-tier downlink heterogeneous cellular networks," IEEE J. Select. Areas Commun., vol. 30, no. 3, pp. 550-560, Apr. 2012.

[6] B. Blaszczyszyn, M. Karray, and H. Keeler, "Using Poisson processes to model lattice cellular networks," in Proc. 32th Annual IEEE International Conference on Computer Communications (INFOCOM13), Apr. 2013, pp. 773-781.

[7] C.-H. Yu, O. Tirkkonen, K. Doppler, and C. Ribeiro, "On the performance of device-to-device underlay communication with simple power control," in Proc. of IEEE 69th Vehicular Technology Conference (VTC Spring 2009), Apr. 2009, pp. 1-5.

[8] Z.-S. Syu and C.-H. Lee, "Spatial constraints of device-to-device communications," in 2013 First International Black Sea Conference on Communications and Networking (BlackSeaCom), Jul. 2013, pp. 94-98.

[9] Z. Liu, T. Peng, Q. Lu, and W. Wang, "Transmission capacity of d2d communication under heterogeneous networks with dual bands," in 7th International ICST Conference on Cognitive Radio Oriented Wireless Networks and Communications (CROWNCOM), Jun. 2012, pp. 169-174.

[10] X. Lin and J. Andrews, "Optimal spectrum partition and mode selection in device-to-device overlaid cellular networks," in Proc. of IEEE Global Communications Conference (GLOBECOM13), Dec. 2013, pp. 18371842.

[11] X. Lin, J. Andrews, and A. Ghosh, "Spectrum sharing for deviceto-device communication in cellular networks," IEEE Trans. Wireless Commun., vol. 13, no. 12, pp. 6727-6740, Dec. 2014.

[12] H. ElSawy, E. Hossain, and M.-S. Alouini, "Analytical modeling of mode selection and power control for underlay D2D communication in cellular networks,", IEEE Trans. Commun., vol. 62, no. 11, pp. 41474161, Nov. 2014. 